pathways $^{12}$, such as WNT/ $\beta$-catenin, PTEN, MAPK and CDK4/6, these findings will ultimately guide the design of immuno-oncology combinations. The discovery of immunogenic LIMIT in melanoma will likely draw considerable attention for therapeutic implication in cancer immunotherapy. In particular, upregulating the expression of LIMIT through the RNA-guide CRISPR activation system dramatically drove tumour MHC-I expression and sensitised tumours to $\mathrm{T}$ cell-mediated antitumour immune responses, highlighting the therapeutic potential of targeting the LIMIT-GBP-HSF1 axis to improve cancer immunotherapy. Due to the lack of efficient approaches to target lncRNAs in clinical settings, it remains a challenge to streamline the 'bench to bedside' translation of lncRNA-related studies. To ensure the efficacy and safety of the clinical application of targeting lncRNAs in combination with cancer immunotherapy, further studies are also needed to elucidate the role of these lncRNAs in regulating the function of immune cells and to determine the in vivo impact of targeting selected lncRNAs in preclinical models.

Taken together, this study reports the contribution of the LIMIT-GBPs-HSF1 axis in promoting $\mathrm{T}$ cell-mediated antitumour immunity and provides novel insights into the role of tumour intrinsic factors in tumour immune evasion. Future studies are critical for determining whether LIMIT can be used as a biomarker to predict responses to immunotherapy or as a therapeutic target to potentiate immunotherapy in patients with cancer. To identify new lncRNA targets to enhance antitumour immunity, it may also be worthwhile to perform CRISPR-based lncRNA screens and characterise lncRNA-based features in non-responders to cancer immunotherapy.

Jiakai Hou and Weiyi Peng (D) $₫$

Department of Biology and Biochemistry,
University of Houston, Houston, TX, USA.

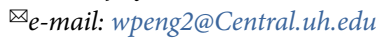

Published online: 8 April 2021

https://doi.org/10.1038/s41556-021-00682-1

References

1. Frankish, A. et al. Nucleic Acids Res. 47, D766-D773 (2019).

2. Schmitt, A. M. \& Chang, H. Y. Cancer Cell 29, 452-463 (2016).

3. Huarte, M. Nat. Med. 21, 1253-1261 (2015).

4. Gaopeng, L. et al. Nat. Cell Biol. https://doi.org/10.1038/s41556021-00672-3 (2021).

5. Hezroni, H. et al. Cell Reports 11, 1110-1122 (2015).

6. Hu, Q. et al. Nat. Immunol. 20, 835-851 (2019).

7. Wang, Q. M., Lian, G. Y., Song, Y., Huang, Y. F. \& Gong, Y. Life Sci. 231, 116335 (2019).

8. Gil, N. \& Ulitsky, I. Nat. Rev. Genet. 21, 102-117 (2020).

9. Chen, Y. G., Satpathy, A. T. \& Chang, H. Y. Nat. Immunol. 18, 962-972 (2017).

10. Zhang, L., Xu, X. \& Su, X. Mol. Cancer 19, 48 (2020).

11. Carpenter, S. et al. Science 341, 789-792 (2013).

12. Kalbasi, A. \& Ribas, A. Nat. Rev. Immunol. 20, 25-39 (2020).

13. Hou, J. et al. J. Immunother. Cancer 9, e001819 (2021).

14. Tawbi, H. et al. J. Clin. Oncol. 36, TPS9596 (2018).

15. Ribas, A. et al. Nat. Commun. 11, 6262 (2020).

Competing interests

The authors declare no competing interests.

\title{
Monocyte and dendritic cell defects in COVID-19
}

Monocytes, plasmacytoid and conventional dendritic cells are crucial for antiviral immune responses. A new study now compares severe and moderate cases of COVID-19 and links defects in viral sensing, interferon and antigen presentation pathways, associated with upregulated apoptosis and inflammatory pathways, to high COVID-19 severity.

\section{Andreas Wack}

ecent studies have implicated

COVID-19 as an infection with complex response patterns that involve many immune cell types. In this issue of Nature Cell Biology, Saichi et al. focused on the analysis of monocyte and dendritic cell (DC) lineages, including conventional (cDCs) and plasmacytoid DCs (pDCs), and showed that multiple pathways for viral sensing, interferon (IFN) signalling, antigen presentation and $\mathrm{pDC}$ maintenance are defective in patients with COVID-19 ${ }^{1}$

(Fig. 1). These findings point towards mechanisms that may explain insufficient control of the viral infection and may open up avenues for therapeutic intervention.

The development of severe COVID-19 in a subset of SARS-CoV-2- infected individuals is linked to both insufficient control of viral replication and excessive inflammation driven by an unbalanced immune response. To better understand the mechanisms underlying severe disease, the authors concentrated on a group of cells called antigen-presenting cells (APCs), comprising myeloid cell populations including monocytes and cDCs, as well as pDCs. These cells were experimentally enriched prior to single-cell RNA sequencing (scRNAseq), allowing a subset analysis at high granularity. All cell types under scrutiny here are potentially at the heart of the action, both in successful and unsuccessful antiviral responses. IFNs are a family of key antiviral cytokines, and most virus-infected host cells can produce and respond to type I IFN (IFN-I). pDCs are known as the IFN factory of the immune system, equipped to detect viruses and able to produce IFN amounts far exceeding those produced by infected cells or other immune cells. This ability of massive amplification of the IFN signal, which also represents a risk for immunopathology, makes it important to understand the fate and functionality of pDCs in COVID-19. Monocytes are intimately associated with the inflammatory phenotypes described for severe COVID-19, as they are both the potential source of inflammatory cytokines and the target of the cytokine storm (for instance, the MHC-II low monocyte phenotype is attributed to high IL-6 levels ${ }^{2-6}$ ). cDCs represent the central link between early innate immune responses 


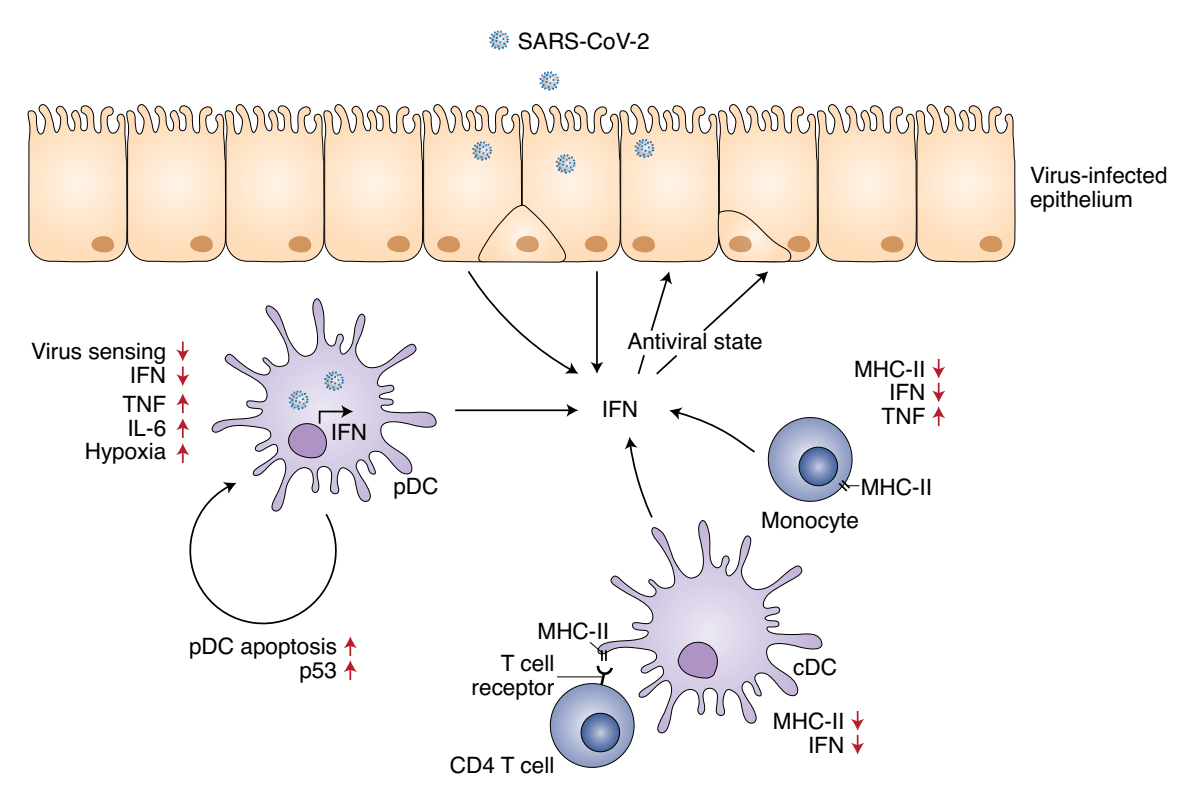

Fig. 1 | Multiple defects in antiviral response pathways found in monocytes and dendritic cells from patients with severe COVID-19. Infection of lung epithelia with SARS-CoV-2 is countered by interferons (IFNs) that signal on infected and adjacent cells to induce antiviral effector mechanisms. IFNs are produced by infected cells, but also by pDCs, cDCs and monocytes. In the present study, lower induction of genes linked to IFN responses were found in patients with severe compared to moderate COVID-19, which may be associated with the reduced levels of genes linked to virus sensing, a prerequisite for IFN induction (red arrows indicate the changes in severe as compared to moderate COVID-19 cases). In contrast, pathways related to pro-inflammatory TNF and NF-kB signalling, hypoxia, IL-6 signalling and others were upregulated in pDCs from severe cases, as were apoptosis-inducing p53 pathways, potentially explaining the loss of pDCs observed in severe COVID-19. In monocytes and various CDC subsets, the same imbalance of increased proinflammatory and reduced IFN pathways as that in pDCs was found. MHC-II molecules are also reduced in these cells, potentially contributing to reduced priming and activation of antiviral T cells in patients with severe COVID-19.

and the adaptive immune response to SARS-CoV-2, due to their unique ability to prime naïve $\mathrm{T}$ cells and thereby trigger cytotoxic $\mathrm{T}$ cell and eventually antibody responses able to specifically eliminate the virus, a crucial step for recovery from COVID-19.

The central requirement for IFN-I in the anti-SARS-Cov-2 response has been clearly shown in studies involving patients with loss-of-function mutations and autoantibodies blocking signals in the IFN-I pathway ${ }^{7,8}$, but not all severe cases of COVID-19 can be explained by this. Here, in their global comparison across all APC subsets between patients with moderate versus severe disease, the authors described that patients with moderate disease showed enrichment in IFN pathways, while patients with severe disease showed less IFN activity and predominantly hypoxia and TNF pathways that were closely associated with inflammation. This points towards a scenario in severe disease in which focused IFN-driven antiviral responses are replaced by inflammatory function of limited antiviral effect but high potential for immunopathology. These findings are in line with other studies suggesting reduced IFN signatures in patients with severe COVID $-19^{9}$, in contrast to the early and strong IFN responses found in successful antiviral responses such as mild SARS-CoV-2 infection ${ }^{10}$. However, it remains to be seen whether a component of the observed divergence in IFN responses may be due to sampling bias. Because IFN responses usually peak early during an antiviral response, it may be that in patients with severe COVID-19, wherein severe symptoms may take longer to develop from the actual infection event than in milder cases, the IFN signature has simply disappeared for this kinetic reason. Longitudinal studies aiming to address this question showed that patients with moderate disease have initially higher IFN-I responses, which wane over time, as is expected in successful antiviral responses, while patients with severe cases started at lower IFN-I levels but showed an increase throughout infection ${ }^{10}$. Similar data are emerging in which influenza and COVID-19

were compared ${ }^{11}$. Here, the course of influenza disease followed the expected sequence of IFNs first and inflammatory cytokines second, but this has not been the case for patients who had COVID-19 with comparable severity: both inflammatory and IFN responses overlapped, pointing to an inappropriate immune response. In the present study, Saichi et al. went on to examine APC subsets to extract cell-type-specific mechanisms at work. Similarly to the global APC analysis, they documented increased inflammatory and pro-apoptotic pathways in pDCs from severe as compared to moderate COVID-19 cases, in combination with reduced IFN-related, immune-sensing and cytotoxic modules, hinting at a profound impairment of pDC survival and function ${ }^{1}$. This is in line with reports on reduced numbers and in vitro activity of pDCs from patients with severe cases ${ }^{2,5,6}$. Severe disease was also linked with a reduced IFN signature and with decreased MHC-II expression when monocytes were assessed in this study, in line with previous findings ${ }^{2,5,12,13}$. This effect is also observed in the inflammatory conditions of severe sepsis and has been linked to excessive IL-6 production ${ }^{3-5}$. In contrast, the higher expression of complement-related genes in monocytes from patients with severe disease may be related to the coagulation phenotype of severe COVID-19. Similar trends towards a reduced IFN signature and MHC class II expression but increased pro-inflammatory pathways were found in the $\mathrm{CDC}$ subsets of patients with severe disease compared to moderate cases.

Together, this high-granularity analysis yields an overall fairly uniform picture of blood APC subsets showing impaired pathways that are central for antiviral protection, namely IFN signatures, viral sensing, antigen presentation and pDC survival (Fig. 1). How well events in the infected lung are represented by measurements on blood immune cells remains to be seen. For instance, immunopathogenic effects of excessive IFNs may also be important in some forms of severe COVID-19 (ref. ${ }^{10}$ ), and these effects may play out locally without much trace systemically, as recently suggested ${ }^{14}$.

Regarding therapeutic options, the results of this study suggest that boosting the pathways found to be impaired in patients with severe disease may be beneficial. IFN therapies have been hotly debated, and several versions have been tested with mixed results and controversial interpretations; this may not come as a surprise, given that both loss of IFN function and excessive IFNs were linked to severe COVID-19 $\left(\right.$ refs. ${ }^{7,8,10}$ ). What seems clear is that IFN 
therapy may be most beneficial if given early, as later intervention may lead to complications due to the pro-inflammatory and anti-proliferative effects associated with IFNs ${ }^{6,10,14,15}$. In addition, whether or not patients with moderate disease should be regarded as prototypes of a successful antiviral response is unclear, as it could be argued that they still require hospital assistance and do not clear the virus with only minimal clinical symptoms, which is the assumed course of infection for many people infected by SARS-CoV-2 who remain below the clinical radar. Through the present study and many others, a coherent picture of severe COVID-19 is slowly emerging, and more work will be required to add higher resolution in time and space to the complex evolution of the immune response and its protective and pathogenic effects in COVID-19.

Andreas Wack (iD)

Immunoregulation Laboratory, Francis Crick Institute, London, UK.

$\bigotimes_{\mathcal{e} \text {-mail: andreas.wack@crick.ac.uk }}$

Published online: 10 May 2021

https://doi.org/10.1038/s41556-021-00685-y

References

1. Saichi, M. et al. Nat. Cell Biol. https://doi.org/10.1038/s41556021-00681-2 (2021).
2. Arunachalam, P. S. et al Science 369, 1210-1220 (2020).

3. Ferreira da Mota, N. V. et al. Shock 50, 293-300 (2018).

4. Giamarellos-Bourboulis, E. J. et al. Cell Host Microbe 27, 992-1000 (2020).

5. Laing, A. G. et al. Nat. Med. 26, 1623-1635 (2020).

$\neg$ 6. Zhou, R. et al. Immunity 53, 864-877.e5 (2020).

7. Zhang, Q. et al. Science 370, eabd4570 (2020).

8. Bastard, P. et al. Science 370, eabd4585 (2020).

9. Hadjadj, J. et al. Science 369, 718-724 (2020).

10. Carvalho, T., Krammer, F. \& Iwasaki, A. Nat. Rev. Immunol. 21, 245-256 (2021)

11. Galani, I. E. et al. Nat. Immunol. 22, 32-40 (2021)

12. Schulte-Schrepping, J. et al. Cell 182, 1419-1440.e23 (2020).

13. Wilk, A. J. et al. Nat. Med. 26, 1070-1076 (2020).

14. Broggi, A. et al. Science 369, 706-712 (2020)

15. Major, J. et al. Science 369, 712-717 (2020).

Competing interests

The author declares no competing interests. 